\title{
Mechanisms of eyewitness suggestibility: tests of the explanatory role hypothesis
}

\author{
Eric J. Rindal ${ }^{1}$ • Quin M. Chrobak $^{2}$ - Maria S. Zaragoza ${ }^{1}$ - Caitlin A. Weihing ${ }^{2}$
}

Published online: 7 February 2017

(C) Psychonomic Society, Inc. 2016

\begin{abstract}
In a recent paper, Chrobak and Zaragoza (Journal of Experimental Psychology: General, 142(3), 827-844, 2013) proposed the explanatory role hypothesis, which posits that the likelihood of developing false memories for postevent suggestions is a function of the explanatory function the suggestion serves. In support of this hypothesis, they provided evidence that participant-witnesses were especially likely to develop false memories for their forced fabrications when their fabrications helped to explain outcomes they had witnessed. In three experiments, we test the generality of the explanatory role hypothesis as a mechanism of eyewitness suggestibility by assessing whether this hypothesis can predict suggestibility errors in (a) situations where the post-event suggestions are provided by the experimenter (as opposed to fabricated by the participant), and (b) across a variety of memory measures and measures of recollective experience. In support of the explanatory role hypothesis, participants were more likely to subsequently freely report (E1) and recollect the suggestions as part of the witnessed event (E2, source test) when the post-event suggestion helped to provide a causal explanation for a witnessed outcome than when it did not serve this explanatory role. Participants were also less likely to recollect the suggestions as part of the witnessed event (on measures of subjective experience) when their explanatory strength had been reduced by the presence of an alternative explanation that could explain the same outcome (E3, source test + warning). Collectively, the results provide strong evidence that the
\end{abstract}

Eric J. Rindal

erindal@kent.edu

1 Department of Psychological Sciences, Kent State University, P.O. Box 5190, Kent, OH 44242, USA

2 University of Wisconsin Oshkosh, Oshkosh, WI, USA search for explanatory coherence influences people's tendency to misremember witnessing events that were only suggested to them.

Keywords Eyewitness suggestibility $\cdot$ False memory Explanatory role $\cdot$ Explanatory coherence

Understanding the causal relationships between events is a fundamental aspect of human cognition. People are highly motivated to achieve a coherent understanding of the world around them (e.g., Schank \& Abelson, 1977), and to this end, they seek causal explanations for people's behavior and the events they experience (e.g., Weiner, 1985). Recent evidence shows that the search for explanatory coherence also plays a role in the memory errors that result from suggestive forensic interviews involving forced fabrication (Chrobak \& Zaragoza, 2013). Forced fabrication refers to situations where witnesses are pressed to provide answers to questions about items/events that they do not remember or never actually experienced. Laboratory studies have shown that this type of coercive interviewing can lead participants to develop false memories for items and even entire events that they had earlier been pressed to fabricate (Ackil \& Zaragoza, 1998, 2011; Chrobak, Rindal, \& Zaragoza, 2015; Chrobak \& Zaragoza, 2008, 2013; Hanba \& Zaragoza, 2007; Memon, Zaragoza, Clifford, \& Kidd, 2010; Zaragoza, Payment, Ackil, Drivdahl, \& Beck, 2001). To illustrate, consider the following interview conducted by an experimenter $(E)$ with a participant $(P)$ who had witnessed a film clip containing a scene where two camp counselors sneak out at night in canoes and receive a severe reprimand from the camp director the next day. During the post-event interview 1 week later, participants were pressed to answer the question, "Where did they (counselors) go and what did they do that caused them to get 
in so much trouble the next day?" despite the clip never depicting where they went. They were informed that they were required to answer all questions, even if they had to guess. Hence, to comply, participants had to make up, or fabricate, a fictitious event:

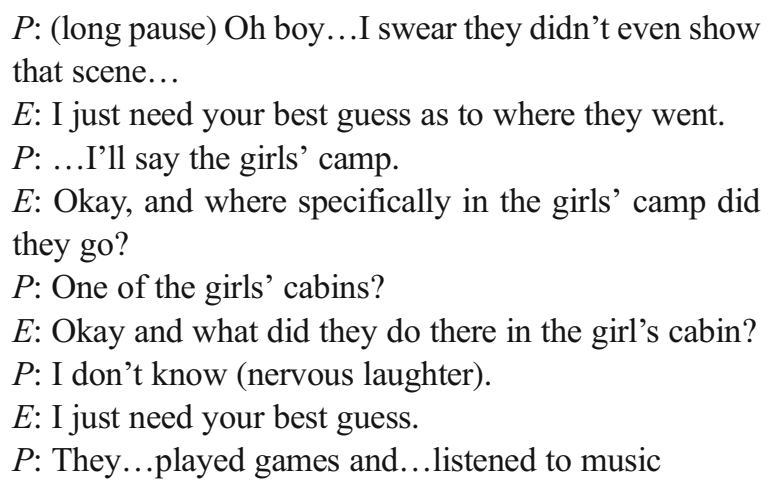

The finding of interest was that 6-8 weeks later, participants incorporated their forced fabrications (e.g., they went to the girls' camp) into their freely provided accounts of the witnessed event almost half the time even when they had correctly and publicly rejected their fabricated event as "not seen" on a 1-week recognition test (Chrobak \& Zaragoza, 2008).

Given that participants were at one time fully aware that these fictitious events were fabrications, why did participants freely report their forced fabrications at such a high rate on a delayed free recall test? Although participants must have, over time, forgotten that they had been forced to fabricate these events, Chrobak and Zaragoza (2013) proposed that a second factor also contributed to false memory development. Drawing on research and theory from the event comprehension literature, they proposed the explanatory role hypothesis, which posits that the likelihood of developing false memories for post-event suggestions is a function of the causal, explanatory role they serve (Chrobak \& Zaragoza, 2013). By this account, participants were especially prone to developing false memories for their forced fabrications, because incorporating their fabrications (e.g., going to the girls' camp) into their mental model of the witnessed event resulted in a better specified, more complete causal explanation for events that they had witnessed (e.g., why they snuck out and got in so much trouble the next day).

To be clear, it was not the case that there were glaring causal gaps in the events participants witnessed that required explanation. The events depicted in the movie (i.e., sneaking out) provided a minimally sufficient explanation for the outcome (i.e., getting in trouble), and the accounts participants fabricated (e.g., what the counselors did and where they went) were not required for the witnessed events to make sense. Many participants provided coherent accounts of the events that they had witnessed without incorporating their forced fabrications (e.g., "They snuck out at night and got in trouble the next day."). In addition, control participants (who did not undergo the forced fabrication interview) very rarely spontaneously inferred causal explanations similar to those that experimental participants were forced to fabricate, thus showing that these errors are not schematic intrusions (Chrobak \& Zaragoza, 2008, 2013). To the contrary, only those participants who were forced to fabricate these more detailed explanations incorporated such events into their eyewitness accounts.

Hence, it does not appear that participants falsely incorporated their forced fabrications into their accounts, because the witnessed events contained causal gaps that required additional explanation. Rather, participants were prone to incorporating their fabrications into their eyewitness accounts, because doing so resulted in richer, more complete, causal explanations of the events that they had witnessed. For example, the events participants were forced to fabricate (e.g., going to the girls' camp to play games and listen to music) provided further insight into the motivations and intentions of the characters (e.g., why they snuck out at night in the first place; what they wanted to do there), thus establishing these motivations as additional causes for the events that they witnessed. Hence, by incorporating their forcibly fabricated events into their memories, participants created a better-specified explanation of how the motivations, intentions, and behaviors of the characters contributed to the witnessed outcomes (cf., Zwaan \& Radvansky, 1998; see Chrobak \& Zaragoza, 2013 for additional discussion).

In support of the explanatory role hypothesis, Chrobak and Zaragoza (2013) provided evidence that when the explanatory role of the fabricated event was diminished (e.g., because it no longer explained a witnessed outcome or because an alternative explanation for the witnessed outcome was provided), the incidence of false memory for fabricated events declined (although it was not eliminated). Hence, the Chrobak and Zaragoza (2013) findings provide the first evidence that the search for explanatory coherence is one goal that can serve as a catalyst to the errors that result from suggestive forensic interviews.

Although the suggestibility of eyewitness memory has been a very active research area, there has been almost no research on the role of the witnesses' goals and motivations in their susceptibility to suggestion. Rather, much of this work has focused on identifying the memory characteristics that lead people to confuse a fabricated or suggested memory with their "real" memory. For example, there is considerable evidence that memories of fabricated or suggested events that are highly familiar, clear, vivid, and rich in sensory and emotional detail are especially likely to become false memories (for reviews see Johnson, Hashtroudi, \& Lindsay, 1993; Lindsay, 2008; Zaragoza, Belli, \& Payment, 2006), especially under conditions that impair retrieval of source specifying 
information (e.g., long retention intervals, limited processing resources). Whereas the foregoing mechanisms have been well-studied, there has been little systematic work examining how the meaning of the suggested information and its relationship to the storyline of the witnessed event might influence witnesses' susceptibility to the suggestion. Relatedly, we know very little about the goals and motivations that might lead witnesses to accept one suggestion as witnessed while rejecting a similar suggestion as not witnessed. Hence, the novel contribution of the explanatory role hypothesis is that it identifies the search for explanatory coherence as one goal that can predispose witnesses to develop false memories for causally relevant post-event information.

\section{Current study}

In many situations where suggestive interview practices are of concern, the interviewer merely provides the witness with false or misleading suggestions (e.g., through leading questions, presuppositions) rather than eliciting false information from the witness. The goal of the current study was to further test the generality of the explanatory role hypothesis as a mechanism of eyewitness suggestibility by assessing whether this hypothesis can predict suggestibility errors in situations where the post-event suggestions are provided by the experimenter (as opposed to fabricated by the participant). In addition, given that all prior evidence bearing on the explanatory role hypothesis has used measures of free recall and recognition, in the current study we assessed whether the explanatory role hypothesis can predict false memory errors when more stringent tests of false memory are used, namely source recognition tests and measures of recollective experience, as well as situations where participants are warned that they have been misled.

The explanatory role hypothesis is built on the premise that people are motivated to enhance their causal understanding of the events they witnessed, and for this reason are especially likely to misattribute post-event information that is causally relevant to the witnessed event. ${ }^{1}$ However, the available evidence bearing on this hypothesis is restricted to studies where participants were asked to fabricate a more complete explanation for a witnessed outcome (Chrobak \& Zaragoza, 2013). This is a potentially important limitation that raises questions about the status of the explanatory role hypothesis as a general mechanism of eyewitness suggestibility. Perhaps asking participants to fabricate these explanations (e.g., "Where did they go, and what did they do that caused them to get in so much trouble?") alters their sensitivity to causally relevant

\footnotetext{
${ }^{1}$ By "explanatory role" we mean that the information provides a causal explanation for an outcome. Hence, we use the terms "causal" and "explanatory" interchangeably, throughout.
}

information. For example, it is possible that asking participants to fabricate these explanations heightens their awareness that their causal understanding is limited. This enhanced awareness of their incomplete causal understanding may increase the likelihood that participants will incorporate their explanatory fabrications into their representation of the witnessed event. Hence, it is possible that Chrobak and Zaragoza's (2013) findings reflect the consequences of asking participants to provide a fuller explanation for witnessed events rather than people's drive for explanatory coherence.

If, as the explanatory role hypothesis posits, people are driven to achieve explanatory coherence, then it should not matter whether participants are asked to generate a more complete causal explanation or merely are presented with a more complete causal explanation of witnessed events. People should, in both cases, be especially likely to update their mental model with post-event sources of information that enhance the explanatory coherence of the witnessed event. To assess the validity of the explanatory role hypothesis as a general mechanism of eyewitness suggestibility, it is important to determine whether the explanatory role hypothesis predicts susceptibility to misinformation even in situations where a causal explanation merely is suggested to participants.

In three experiments, participants watched a video clip (the witnessed event) and subsequently completed a questionnaire that presupposed a fictitious event that participants had not witnessed. One week later, participants were tested on their memory for the witnessed event. The materials and procedures employed in the current experiments were nearly identical to Chrobak and Zaragoza (2013) with the exception that in all experiments the post-event suggestion was provided (rather than fabricated) and we employed a much shorter retention interval ( 1 week rather than 6 weeks). In addition, in Experiments 2 and 3 we employed a more stringent test of false memory development: a source recognition test with measures of recollective experience (rather than recognition and free recall), and in Experiment 3 we also warned participants that they had been misled. The question of interest in all experiments was whether false claims of having witnessed the suggested event would be a function of the suggestion's explanatory role.

\section{Experiment 1}

In Experiment 1, the primary manipulation involved the relationship between the suggested event and the originally witnessed event. In the Explanatory group, the suggested event helped to explain a negative outcome that had been witnessed in the video, but in the Non-Explanatory group the same suggested event did not. Rather, in the NonExplanatory group, video was edited so there was no witnessed outcome that the suggested event helped to explain. 
Participants were asked to freely recall the witnessed event 1 week later. The explanatory role hypothesis predicts that participants in the Explanatory group should be more likely to incorporate the suggested event into their eyewitness accounts than those in the Non-Explanatory group, as the suggested event explains a witnessed outcome in the Explanatory group only. ${ }^{2}$

\section{Method}

Participants Ninety-two (54 females) undergraduates completed the experiment in partial fulfillment of a course requirement. Of these, 50 participants were in the Explanatory group and 42 were in the Non-Explanatory group. ${ }^{3}$

Phase 1: eyewitness event Participants were informed that they would view a video and later be asked questions about the video. Groups of up to six participants viewed one of two edited versions of an 18-minute movie clip that depicts the adventures of Delaney, a counselor at a summer camp (Chrobak \& Zaragoza, 2013). In one scene, Delaney and a fellow counselor are seen sneaking out at night in a stolen canoe. For all participants, the movie clip then cuts to the next day and does not depict where the counselors went or what they did after getting into the canoe.

The experimental manipulation occurred in the scene immediately following the counselors sneaking out. In the Explanatory group, the scene showed Delaney receiving a severe reprimand from the camp director, and in the NonExplanatory group the reprimand scene was replaced with a scene depicting Delaney horseback riding (Fig. 1). Except for the reprimand/horseback riding scene, the movie clips in the two groups were identical.

Phase 2: suggested misinformation via post-event questionnaires Following a 30-minute filler task, all participants received an identical 6-item written questionnaire, which queried participants about salient scenes from the video. The fifth item on the questionnaire presupposed one piece of suggested

\footnotetext{
${ }^{2}$ We did not include a control condition where participants were not exposed to the misleading false information prior to test, because prior studies using similar materials and procedures have consistently shown that control participants who are not misled rarely spontaneously report these items (Chrobak \& Zaragoza, 2008, 2013).

${ }^{3}$ In Experiments 1 and 2, the data was collected at two Midwestern universities concurrently, with approximately half the participants from each site $N$ 's = 55 and 39 for E1, and N's = 30 and 54 for Experiment 2. In both experiments, performance did not vary, nor interact, with research site. Hence, all results are reported collapsed across research site. Although the current study employed experimenter provided misinformation rather than forced fabrication, for each group in Experiments 1 and 2, we sought to collect a sample that was comparable in size to that used in prior forced fabrication studies with these materials and a one-week retention interval (Chrobak \& Zaragoza, 2008).
}

misinformation (in italics below) in the orienting statement and was unrelated to the question participants had to answer.

"Towards the end of the movie, Delaney and a friend use a boat to sneak off at night and go toilet paper the Chief's cabin, causing them to get in trouble. Describe everything you remember about the boat. For example, what type of boat was it and where did they get it from?"

Neither version of the video showed or mentioned anyone toilet papering a cabin, nor was the Chief's cabin depicted. Hence, the suggestion was blatantly false. As depicted in Fig. 1, for participants in the Explanatory group, the suggested event (i.e., toilet papering the Chief's cabin) served an explanatory function, because it helped to explain why Delaney was reprimanded by the Chief (the scene witnessed immediately after the boys snuck out). However, for participants in the Non-Explanatory group, the same suggested event (i.e., toilet papering the cabin) did not serve an explanatory function, because it was unrelated to Delaney horseback riding (the scene that followed the boys sneaking out in the NonExplanatory group).

Phase 3: measure of false memory for suggestions-free recall of witnessed event One week later, participants returned and were instructed to report the details of the video as accurately and in as much detail as possible, as if they were eyewitnesses whose testimony could be used in court. Following this instruction, participants were free to report as much or as little as they wished and were not prompted any further.

\section{Results}

Two blind raters coded all free recall transcripts for clear, uncontroverted reports of the suggested event (i.e., toilet papering the cabin). Generic statements about the counselors going somewhere/doing something or qualified reports of the toilet papering event (e.g., "but I'm not sure I saw that") were not counted. Inter-rater reliability, or the percent total agreement, was $98 \%$. Disagreements were resolved by a third blind rater.

As illustrated in Fig. 2, the results are consistent with the predictions of the explanatory role hypothesis: Participants were significantly more likely to falsely report the suggested event when that event explained the witnessed outcome (Explanatory group) than when the same suggestion did not help to explain a witnessed outcome (Non-Explanatory group), $X^{2}(1, N=92)=7.06, p<0.01, \varphi=0.27$. $^{4}$

\footnotetext{
${ }^{4}$ Participants in the Non-Explanatory group read in the narrative that the counselors got in trouble even though they had not witnessed that scene, and so "getting in trouble" was a second piece of misinformation for them. However, no participants in the Non-Explanatory group freely reported that the counselors got in trouble at the time of the final test.
} 

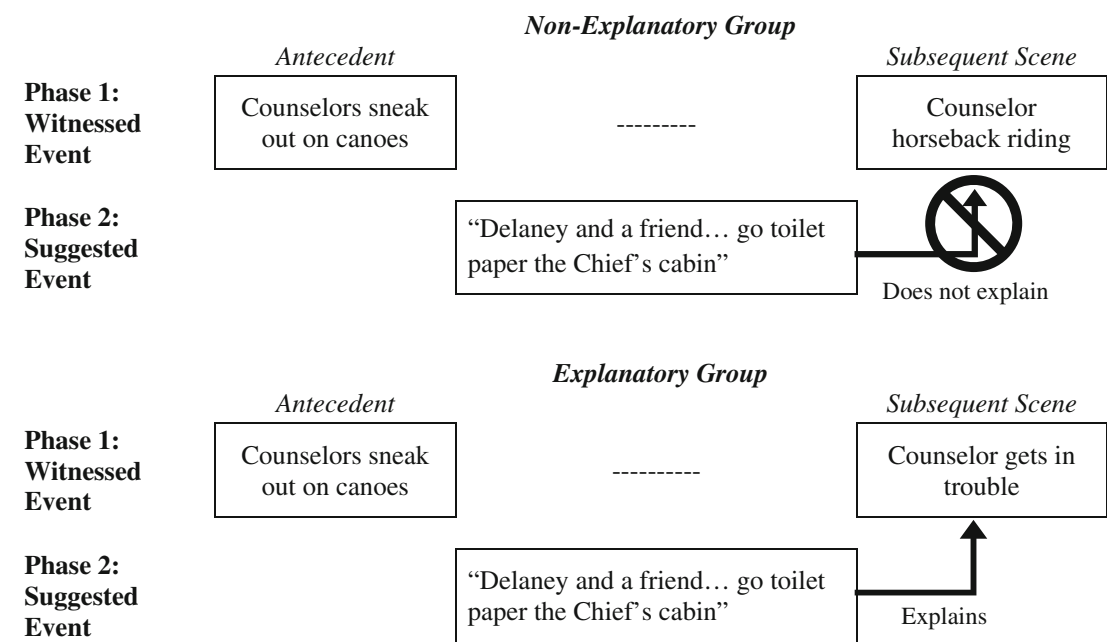

Fig. 1 Overview of procedure and explanatory role manipulation for Experiments 1 and 2

\section{Discussion}

Although the suggestive manipulation employed in Experiment 1 was different from that employed by Chrobak and Zaragoza (2013), the finding was the same: false reporting of the post-event suggestion was a function of the explanatory relationship between the suggestion and the previously witnessed event. These results establish that Chrobak and Zaragoza's (2013) findings generalize to situations where post-event suggestions are read, rather than generated.

Although the results of Experiment 1 are consistent with the predictions of the explanatory role hypothesis, they do not provide conclusive evidence that false memory rates were higher in the Explanatory group. An alternative possibility is that participants in both groups developed false memories of the suggested event to the same extent, but participants in the Explanatory group were simply more likely to report them. Given that narrative recall is structured around the causal and logical sequence of events, it is possible that these results reflect participants' tendency to report preferentially the

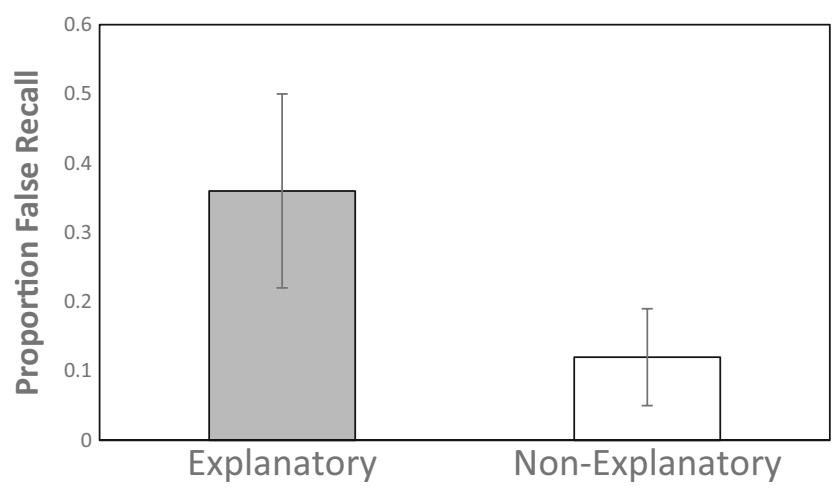

Fig. 2 Mean proportion of participants in the Explanatory and NonExplanatory groups of Experiment 1 who reported the suggested information 1 week later on the final recall test. Error bars represent 95\% confidence intervals events that fit within a causal sequence. As illustrated in Fig. 1, in the Explanatory group, the suggestion can be integrated into a causal chain of witnessed events, but in the NonExplanatory group it is not possible to do so, because the suggestion was not linked to any witnessed consequence (cf., Chrobak \& Zaragoza, 2013).

In Experiment 2, we sought to obtain clearer evidence that a suggestion's explanatory role is directly linked to false memory development. To this end, at the time of the final test, participants in both groups were presented with the suggested event on a source recognition test, where they were asked to make judgments about each item's source (i.e., from the video or questionnaire). Of interest was whether participants would be more likely to misattribute the suggested event to the video when it served an explanatory function relative to when it did not. In those cases, where participants falsely attributed the suggestion to the video, we further directed them to indicate whether they specifically remembered seeing the suggested event in the video or whether they simply believed that the suggested event was in the video. The explanatory role hypothesis predicts that, relative to the Non-Explanatory group, participants in the Explanatory group should be more likely to falsely "remember" seeing the suggested event in the video.

\section{Experiment 2}

\section{Method}

Participants A total of 84 (44 females) undergraduates completed the experiment in partial fulfillment of a course requirement, with 43 in the Explanatory group and 41 in the NonExplanatory group.

The materials and procedure were identical to Experiment 1 with the exception that the final test was a source memory test. 
Phase 3: measure of false memory for suggestions-source recognition test Participants heard a series of 16 statements and were asked to answer 2 questions for each: (1) whether they remembered it from the video, and (2) whether they remembered it from the questionnaire. Note that these source judgments were not mutually exclusive, and participants could indicate that the test item came from one source, both sources, or neither source. For each of these questions, there were three response options: "remember," "believe," or "neither." Participants were instructed to select "remember" only if they could consciously recollect that the test item came from that source and to select "believe" if they were not able to consciously recollect the original experience but believed that the test item was from that source. The instructions emphasized that "remember and "believe" did not correspond to high and low confidence, and subjects were given examples of situations where one might have high confidence something happened, despite the absence of a conscious recollection.

The test statements consisted of the suggested event (which was only in the questionnaire) intermixed with 15 filler items from 3 other sources ( 5 video only, 5 both video and questionnaire, and 5 new). Prior to taking the test, participants were correctly informed that the test contained statements from each of the four possible source categories.

Given that the goal of this study was to assess false memory for suggested misinformation, we present results for the suggested event only (we note that there were no statistically significant group differences on filler item performance, all $p s>0.10$ ).

\section{Results}

Table 1 shows the mean proportion of "remember," believe," and "neither" responses to each of the two source probes as a function of group. The results support the explanatory role hypothesis: Relative to the Non-Explanatory group, participants in the Explanatory group were more likely to misattribute the suggested event to the video (mean total "remember" + "believe" in video responses were 0.82 versus 0.39 for the

Table 1 Mean proportion of remember, believe, or neither responses to "In Video?" and "In Questions?" for the suggested item in the Explanatory and Non-Explanatory groups of Experiment 2

\begin{tabular}{lll}
\hline & Explanatory & Non-Explanatory \\
\hline In video? & & \\
$\quad$ Remember & 0.56 & 0.10 \\
Believe & 0.26 & 0.29 \\
Neither & 0.19 & 0.61 \\
In questions? & & \\
Remember & 0.37 & 0.10 \\
Believe & 0.35 & 0.15 \\
Neither & 0.28 & 0.76 \\
\hline
\end{tabular}

Explanatory and Non-Explanatory groups, respectively, $\left.X^{2}(1, N=84)=15.80, p<0.001, \varphi=0.43\right)$ and more likely to "remember" incorrectly the suggested item in the video $(M=0.56$ vs. $M=0.10), X^{2}(1, N=84)=20.04, p<0.001$, $\varphi=0.49$.

Although suggestions that served an explanatory role were more often incorrectly misattributed to the video, these same suggestions were more often accurately attributed to the questionnaire relative to those not serving an explanatory role. Participants in the Explanatory group were more likely than the Non-Explanatory group to attribute correctly the suggested item to the questionnaire (mean total "remember" + "believe" in questions responses were 0.72 and 0.25 for the Explanatory and Non-Explanatory groups, respectively, $X^{2}(1$, $N=84)=19.11, p<0.001, \varphi=0.48$ ) and more likely to "remember" that the suggestion from the questionnaire $(M=0.37$ vs. $M=0.10), X^{2}(1, N=84)=8.72, p<0.01, \varphi=0.32$.

The foregoing analysis cannot discern how participants distributed their responses to the two source probes (i.e., what proportion indicated that the suggestion was from the video only, questionnaire only, both sources, or neither). Inspection of Table 2 reveals that there were large group differences in the proportion of participants who had no memory of the suggestion at all: Whereas participants in the Non-Explanatory group often endorsed that the suggested event was from neither source, participants in Explanatory group rarely did so $\left(X^{2}(1\right.$, $N=84)=20.13, p<0.001, \varphi=0.49)$. Because participants cannot make source judgments about events they do not remember, it is possible that the group differences in source memory reported above are a consequence of the differences in item memory as opposed to differences in source memory. To control for differences in item memory, we next report source judgments for only those suggestions that participants recognized as "old" (i.e., identified in at least one source).

As illustrated in Fig. 3, conditionalizing the data on recognized suggestions does not change the pattern of results. Participants in the Explanatory group were more likely than those in the Non-Explanatory group to incorrectly claim that they "remembered" witnessing the suggestion in the video (M's $=0.60$ and 0.20 for the Explanatory and Non-Explanatory groups, respectively, $X^{2}(1, N=60)=8.57$, $p=0.003, \varphi=0.38)$, although group differences in total

Table 2 Joint probabilities of yes (remember + believe) and no (neither) responses to "in Video?" and "In Questions?" for the suggested item in the Explanatory and Non-Explanatory groups of Experiment 2

\begin{tabular}{|c|c|c|c|c|c|}
\hline \multicolumn{3}{|c|}{ Explanatory } & \multicolumn{3}{|c|}{ Non-Explanatory } \\
\hline \multirow[b]{2}{*}{ Questions } & \multicolumn{2}{|c|}{ Video } & \multirow[b]{2}{*}{ Questions } & \multicolumn{2}{|c|}{ Video } \\
\hline & Yes & No & & Yes & No \\
\hline Yes & 0.60 & 0.12 & Yes & 0.15 & 0.10 \\
\hline No & 0.21 & 0.07 & No & 0.24 & 0.51 \\
\hline
\end{tabular}




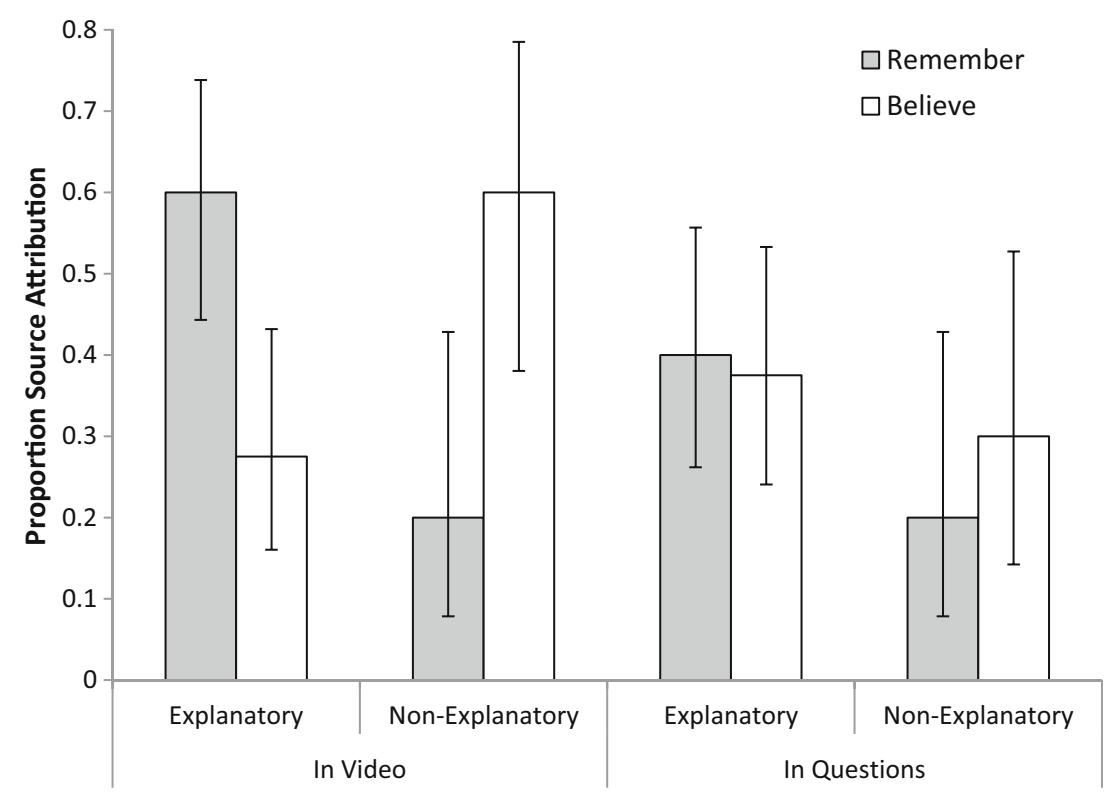

Fig. 3 Mean proportion of recognized suggestions participants claimed to (a) remember from the video, (b) believe was in the video, (c) remember was in the written materials, and (d) believe was in the

misattributions ("remember" + "believe") were no longer statistically reliable (M's $=0.88$ vs. $0.80, X^{2}(1, N=60)=0.59$, $p=0.44)$. Similarly, when examining total accurate attributions to the questionnaire ("remember" + "believe" in questionnaire), participants in the Explanatory group were more likely than those in the Non-Explanatory group to indicate that the suggested event was from the questionnaire (M's $=0.78$ vs. $0.50, X^{2}(1$, $N=60)=4.66, p=0.03, \varphi=0.28$ ), although group differences in "remember" in questionnaire responses were not reliable (M's $=0.40$ vs. $0.20, X^{2}(1, N=60)=2.40, p=0.12$ ).

\section{Discussion}

The present findings extend those of Chrobak and Zaragoza (2013) by showing that suggestions that serve an explanatory role are more likely to develop into false recollections of having witnessed the suggested event in the video. It also was the case that suggested events that served an explanatory function were much better remembered than those that did not. When the suggested event did not serve an explanatory function, a large proportion of participants forgot the suggested event after just 1 week. A likely reason for these large group differences in memory for the suggestion is that the suggestions in the Explanatory group were linked to both an observed antecedent and an observed consequence, but those in the NonExplanatory group were linked to an observed antecedent only. This resulted in a sparser number of connections between the suggested and witnessed event in the Non-Explanatory group relative to the Explanatory group.

Given that there were a greater number of connections between the suggested and witnessed event in the written materials, presented for the Explanatory and Non- Explanatory groups of Experiment 2. Error bars represent 95\% confidence intervals

Explanatory group (relative to the Non-Explanatory), what is unclear from the results of Experiments 1 and 2 is the extent to which greater false memory in the Explanatory group is due to the explanatory nature of these connections. Although explanatory role and interconnectivity often co-vary, in Experiment 3 we sought to assess whether the explanatory role of a suggestion contributes to false memory development independent of interconnectivity with the witnessed event.

\section{Experiment 3}

Experiment 3 employed a different approach to evaluating the explanatory role hypothesis. In Experiment 3, all participants were in the Explanatory group; hence, for all participants, the suggested event was linked to an outcome they had witnessed (Fig. 4). What varied was whether participants were provided with a possible alternative explanation for the witnessed outcome that was explained by the post-event suggestion. Research on causal reasoning has shown that the strength of a perceived causal relationship is influenced by the presence of alternative explanations, such that having multiple possible explanations reduces the extent to which people view any one event as causally related to that outcome (Einhorn \& Hogarth, 1986; Kelley, 1973). By the same token, having only one causal explanation for an event increases the perceived strength of the causal relationship. Hence, the presence of a potential alternative explanation for the witnessed outcome should reduce the explanatory strength of the suggested event (Low Explanatory strength) relative to situations where there is no alternative explanation for the witnessed event explained 

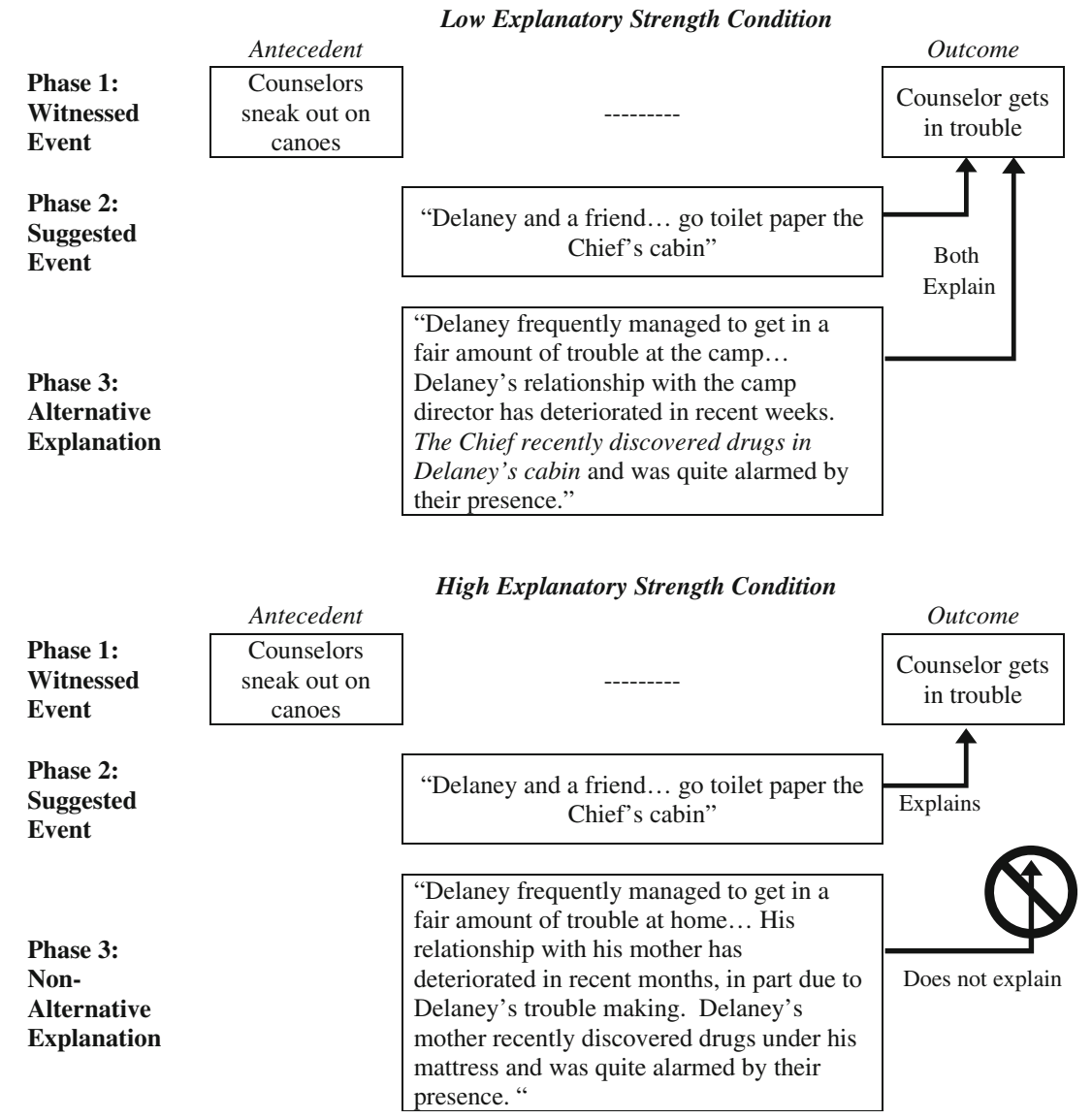

Fig. 4 Overview of procedure and explanatory strength manipulation for Experiment 3

by the suggestion (High Explanatory strength). According to the explanatory role hypothesis, false memory should be a function of the explanatory strength of the suggestion, such that reducing the explanatory strength of a suggested event (through the presence of a potential alternative explanation) should result in lower false memory for that suggestion.

A second change introduced in Experiment 3 was explicitly warning participants immediately before the final test that the post-event questionnaire contained information that they had never witnessed. Although the source recognition test informed participants that some of the test items were from the post-event questionnaire only, in Experiment 3 we also explicitly warned participants that the questionnaire was discrepant with what they had witnessed and that their task was to distinguish between those events they had witnessed and the information encountered in the questionnaire. Prior studies with similar materials have documented that such warnings reduce the incidence of false memory errors, even when source recognition tests are used (e.g., Ackil \& Zaragoza, 2011; see Blank \& Launay, 2014 for recent a meta-analysis of warning effects). By testing the explanatory role hypothesis under conditions that are effective at reducing false memory errors, we sought to establish the generality of the explanatory role hypothesis.

\section{Method}

Participants A total of 140 (70 females, 14 not reported) undergraduates completed the experiment in partial fulfillment of a course requirement. Sample size was based on an a-priori power analysis ( $\mathrm{G}^{*}$ Power 3; Faul, Erdfelder, Lang, \& Buchner, 2007) to detect a medium effect size ( $w=0.24, \alpha=$ $0.05,1-\beta=0.80, \mathrm{df}=1$ ), which indicated a necessary sample of 137 participants. ${ }^{5}$

Unless otherwise noted below, the materials and procedure were identical to those of the Explanatory group of Experiment 2. The primary innovation was the introduction of a second source of background information about the

\footnotetext{
${ }^{5}$ A larger sample was employed in Experiment 3 (relative to Experiments 1 and 2), for two reasons. First, the manipulation of the suggestion's explanatory role in Experiment 3 is much subtler (higher explanatory role strength vs. lower explanatory role strength) than in the foregoing experiments (explanatory role vs. no explanatory role), and we expected a much smaller effect size as a consequence. Second, we expected that the overall rate of false memory would be suppressed as a consequence of including a pre-test warning, making it more difficult to detect potential differences in false memory rates as a function of the suggestions' explanatory strength. Thus, the power analysis was based on an effect size from Experiment 3 of Chrobak and Zaragoza (2013) that utilized a similar manipulation of explanatory strength.
} 
characters from the video that introduced potential alternative explanations for the witnessed outcomes (Fig. 4).

Phase 1: eyewitness event Only the video employed in the Explanatory conditions of Experiments 1 and 2 was used. Hence, for all participants, the post-event suggestions explained a witnessed outcome.

Phase 2: suggested misinformation via post-event questionnaires An additional question was added to the postevent questionnaire for purposes of introducing a second piece of suggested post-event misinformation that served an explanatory role. The new question referred to a scene in the video where Delaney stands up to make an announcement, loses his balance, and falls on the floor. Item 3 on the questionnaire introduced the explanatory suggestion that Delaney fell, because Ratface (a camper) tied his shoelaces together:

"The next scene takes place in the dining hall. Delaney is asked to stand up to give an announcement. As a practical joke, Ratface tied Delaney's shoelaces together, causing him to end up on the floor. Give a detailed description of the dining hall. What did it look like and who was there?"

This suggestion is blatantly false because the video did not depict Ratface (or anyone else) coming anywhere near Delaney in this scene, much less tying his shoelaces together.

Phase 3: alternative explanation manipulation - character vignettes Immediately after completing the post-event questionnaire, participants were asked to read three vignettes (4 paragraphs each) that provided background information about the lives of three characters from the movie clip (cf., Chrobak \& Zaragoza, 2013). The information contained in the vignettes was not suggested misinformation, because the vignettes did not contradict what participants had witnessed but instead provided information that went beyond the events they had witnessed. The experimental manipulation occurred in a vignette about Delaney.

All participants had earlier received the misleading suggestion that Delaney fell, because Ratface tied his shoelaces together. In paragraph two of the Delaney vignette, all participants also learned that Delaney suffered from a medical disorder. Half of the participants learned that he had Meniere's disease, a rare inner ear disorder that causes unexpected periods of vertigo and dizziness, thus providing a potential explanation for why Delaney fell in the dining hall (although the vignette never made this connection). Having a potential alternative explanation for Delaney's fall lowered the explanatory strength of the suggestion that his fall was a consequence of Ratface tying his shoelaces (Low Explanatory Strength condition). The remaining participants read instead that Delaney suffered from a rare skin disorder known as Pemphigus, which produced blisters on his face, neck, and back. For these participants, the disease provided no explanation why Delaney fell in the dining hall and thus the suggestion (i.e., Ratface tying his shoelaces) remained the sole explanation for the fall (High Explanatory Strength condition).

All participants had earlier received a second suggestion: that Delaney was reprimanded by the Chief, because he had toilet-papered the Chief's cabin. In paragraph four of the Delaney vignette, all participants also learned that Delaney had gotten in trouble for having drugs in his possession. As illustrated in Fig. 4, half of the participants learned that the Chief had found drugs in Delaney's cabin, thus providing a possible alternative explanation why Delaney was reprimanded by the Chief and lowering the explanatory strength of the suggestion (Low Explanatory Strength condition). The remaining participants learned that Delaney's mother had found drugs in his bedroom long before he went to camp. For these participants, the drug possession does not explain why he was reprimanded by the Chief at camp, and the suggestion remained the sole explanation (High Explanatory Strength condition; Fig. 4).

The explanatory strength of the suggested events was manipulated within subjects, such that for all participants one of the suggestions served in the Low Explanatory strength condition and the other suggestion served in the High Explanatory strength condition, with materials counterbalanced across the experiment.

Phase 4: measure of false memory for suggestions - source recognition with warning The source test was identical to that employed in Experiment 2, with the exception that four test items were added: the second suggested event (i.e., "Ratface tied Delaney's shoe laces together") that was in the questionnaire only, and three items from vignettes: the two pieces of information that served as potential alternative/ nonalternative explanations for the witnessed outcome (i.e., "Delaney suffered from a rare medical disorder," and "Delaney kept drugs hidden under a mattress"), and a third filler item.

In addition, before receiving the source recognition test instructions, participants also received the following warning: "...when we asked you questions last week, those questions mentioned events that never actually happened in the video. Your task today is to determine what was in the video and what was in the written materials you read." The question of primary interest was whether participants would be more likely to misattribute the suggested events to the video in the High Explanatory Strength condition, relative to the Low Explanatory Strength condition.

\section{Results}

As in Experiment 2, we report misattributions of the suggestions to the video and correct attributions of the suggestions to 
the written materials in two ways: the absolute proportion of source attributions (Table 3 ) and source attributions conditionalized on item recognition (Fig. 5). However, because manipulating the explanatory strength of the suggestion did not lead to reliable differences for item recognition (McNemar's $X^{2}(1, N=140)=0.21, p=0.65$; Table 4), both measures yielded the same pattern of results. For the sake of brevity, we report the results of the conditional analyses only.

As illustrated in Fig. 5, the results supported the explanatory role hypothesis: misattributions of the suggested items to the video were a function of the explanatory strength of the misleading suggestion. Misattributions were greater in the High Explanatory strength condition than in the Low Explanatory strength condition, and this was true for both total misattributions ("remember" + "believe") to the video, (M's $=0.74$ and 0.85 for the Low and High Explanatory strength conditions, respectively, $\left.X^{2}(1, N=230)=4.56, p=0.03, \varphi=0.14\right)$, as well as those misattributions to the video that were "remembered" (M's $=0.42$ and 0.57 for the Low and High Explanatory strength conditions, respectively, $X^{2}(1, N=230)=5.63$, $p=0.018, \varphi=0.16) .{ }^{67}$ Figure 5 also shows that there were no statistically significant differences between the Low and High Explanatory strength conditions in total ("remember" + "believe") attributions to the written materials (M's $=0.59$ vs. 0.61 for the Low and High Explanatory strength conditions respectively, $\left.X^{2}(1, N=230)=0.38, p=0.54\right)$ nor in "remember" attributions to the written materials (M's $=0.25$ vs. 0.33 for the Low and High Explanatory strength conditions respectively, $\left.X^{2}(1, N=230)=2.04, p=0.15\right)$.

Finally, we analyzed the extent to which participants misattributed to the video the relevant information from the vignettes (i.e., Delaney had a rare medical disorder) as a function of explanatory condition. The explanatory role hypothesis predicts that misattributions of the vignette information to the video should be a function of the explanatory role the information serves. For example, participants should be more likely to misattribute "Delaney had a rare medical disorder" to the video if they had earlier read that Delaney had a balance disorder relative to a skin disorder. Consistent with the explanatory role hypothesis, participants were more likely to misattribute the critical vignette items to the video when they provided an alternative explanation for the witnessed outcome (mean total $=0.24$ ) than when they did not serve this explanatory function (mean total $=0.14), X^{2}(1, N=251)=4.38$, $p=0.036, \varphi=0.13)$. No other effects were reliable.

\footnotetext{
${ }^{6}$ The pattern of results was the same for both the prank and sneak critical items: When conditionalized on item recognition, mean "remember in video responses" for the low versus high explanatory strength conditions were 0.54 versus 0.69 and 0.29 versus 0.45 for the prank and sneak items, respectively. For this reason, we report the data collapsed across item.

${ }^{7}$ Because the data were conditionalized on item recognition, there were a number of empty cells. As a result, the data were analyzed by using a test that does not assume correlated samples.
}

Table 3 Mean proportion of remember, believe, or neither responses to "In Video?" and "In Written Materials?" for the suggested item in the High and Low Explanatory Strength conditions of Experiment 3

\begin{tabular}{lll}
\hline & High explanatory & Low explanatory \\
\hline In video? & & \\
$\quad$ Remember & .46 & .35 \\
Believe & .22 & .26 \\
$\quad$ Neither & .31 & .39 \\
In written materials? & & \\
$\quad$ Remember & .20 & .28 \\
Believe & .27 & .24 \\
Neither & .53 & .48 \\
\hline
\end{tabular}

\section{Discussion}

The results provide strong support for the explanatory role hypothesis. When both the number and explanatory nature of the connections between the suggested event and the witnessed event was equated for all participants, false memory development was a function of the suggestion's explanatory strength. As predicted, the availability of a potential alternative to the suggestion as an explanation for the witnessed outcome lowered the explanatory strength of these suggestions and resulted in a decline in false memory. We note that whereas the suggested events were explicitly presented to participants as explanations for the witnessed outcomes, the information that served as potential alternative explanations was not explicitly linked to the events they had witnessed or to the post-event suggestions. Hence, it is clear that participants continued to search for information that was potentially relevant to explaining prior events, even when the new information they encountered was not explicitly linked to the events they witnessed.

Finally, it is noteworthy that the explanatory strength of the suggestions predicted false memory development, even though all the suggestions served an explanatory function. In addition, we observed differential false memory under conditions that have been shown to optimize source monitoring accuracy and minimize false memory errors, namely, source recognition tests accompanied by a warning. The finding that the explanatory strength of the suggestions predicted false memory rates even under these very stringent testing conditions we take as especially convincing support for the explanatory role hypothesis.

\section{General discussion}

The current findings, in combination with those of Chrobak and Zaragoza (2013), provide strong support for the conclusion that a post-event suggestion's value in furthering a 


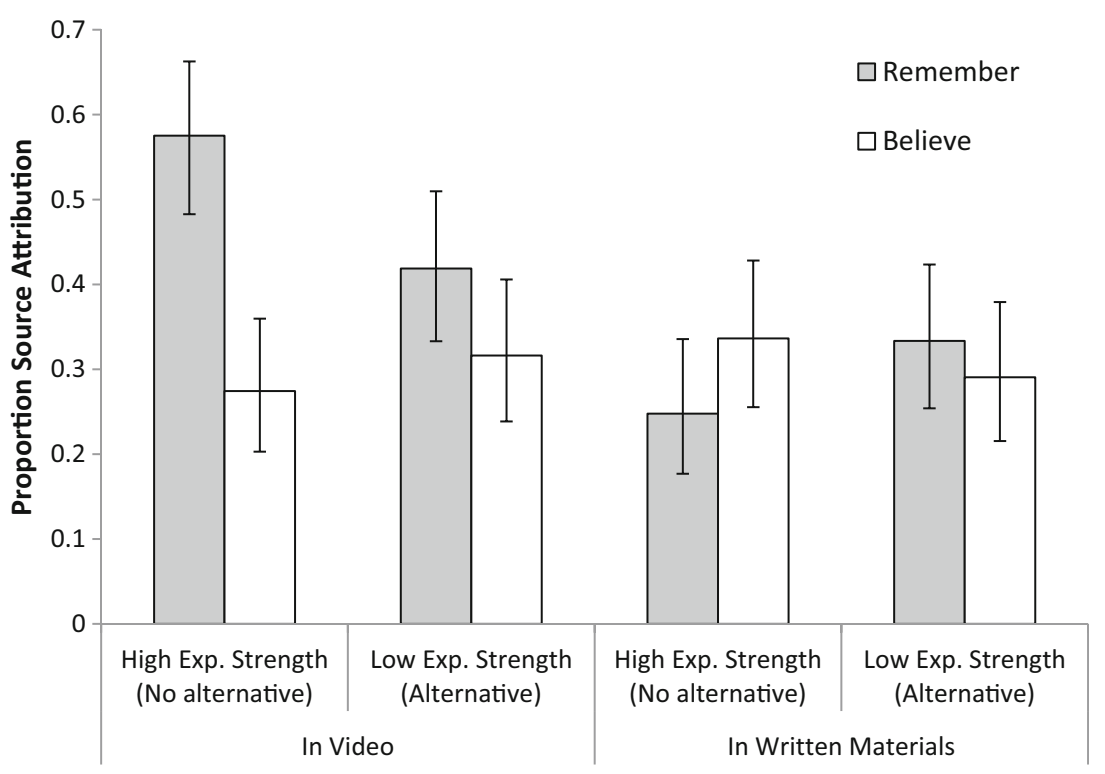

Fig. 5 Mean proportion of recognized suggestions participants claimed to (a) remember from the video, (b) believe was in the video, (c) remember was in the written materials and (d) believe was in the

written material, presented for the High Explanatory Strength and Low Explanatory Strength conditions of Experiment 3. Error bars represent $95 \%$ confidence intervals

person's causal understanding of a witnessed event is a factor that contributes to the development of false memories for the post-event suggestion. The present study extends prior findings by showing that the explanatory role hypothesis predicts false memory development for both post-event suggestions that have been provided to participants as well as those selfgenerated by them. An additional novel contribution of the current study is the finding that the suggestion's explanatory role predicted false recollections of having witnessed the suggested event (not just belief that the suggested event transpired) and did so even when participants were given source recognition tests accompanied by a warning. Previous studies have shown that, relative to traditional recognition tests, source monitoring tests reduce (Zaragoza \& Lane, 1994) and even eliminate (Lindsay \& Johnson, 1989) suggestibility effects, presumably because having to make overt source judgments alerts participants to the fact that there are multiple sources of information about the witnessed event, and the task

Table 4 Joint probabilities of yes (remember + believe) and no (neither) responses to "In Video?" and "In Written Materials?" for the suggested items in the High and Low Explanatory Strength conditions of Experiment 3

\begin{tabular}{|c|c|c|c|c|c|}
\hline \multicolumn{3}{|c|}{ High explanatory strength } & \multicolumn{3}{|c|}{ Low explanatory strength } \\
\hline & Videc & & & Video & \\
\hline Written materials & Yes & No & Written materials & Yes & No \\
\hline Yes & 0.35 & 0.12 & Yes & 0.30 & 0.22 \\
\hline No & 0.34 & 0.19 & No & 0.31 & 0.16 \\
\hline
\end{tabular}

requires that they discriminate between different sources of information in memory. Consequently, when completing a source recognition test participants may retrieve and use source specifying information that they do not access under other test contexts (e.g., recall and recognition), where they may rely on more accessible aspects of a memory, such as familiarity. Hence, our results provide strong evidence that the explanatory role hypothesis not only predicts the false reporting of suggested information, it also predicts the development of genuine false memories.

The present findings fit with the broader literature on text and event comprehension processes, where it has been extensively documented that achieving explanatory coherence is a fundamental aspect of event comprehension processes. For example, theories of narrative text comprehension share the assumption that readers seek to identify the implicit and explicit causes that link the elements of a story together, thereby achieving explanatory coherence (McNamara \& Magliano, 2009, for a comprehensive review; Magliano, Radvansky, \& Copeland, 2007 and Zacks, Speer, \& Reynolds, 2009 for evidence that these same processes underlie witnessed event comprehension). Our research on eyewitness suggestibility phenomena extends these findings by showing that the search for explanatory coherence continues well after participants have witnessed the target event. As noted earlier, the events depicted in the video provided a reasonably coherent causal explanation for the events participants witnessed. Nevertheless, participants in our experiments were especially prone to incorporating post-event suggestions into their memory for the witnessed event when doing so provided a more complete causal explanation for the events they had earlier 
witnessed. Even more surprising was the finding in Experiment 3 that even after participants had encountered the post-event suggestions, they continued to update their memory when they encountered new sources of information that were potentially causally relevant. In Experiment 3, participants evidenced lower false memory for the suggested events when they had been exposed to general background knowledge that provided a potential alternative explanation for the witnessed outcomes. Collectively, these findings show that participants' memory for the witnessed events reflected not what they saw but rather the causal understanding they constructed by updating their memory of the witnessed event with causally relevant information they gathered from multiple post-event sources.

Although the present studies provide strong support for the explanatory role hypothesis, a number of questions remain about the limits and generalizability of the false memory effects reported here. For example, in the current set of experiments (as well as in Chrobak \& Zaragoza, 2013) the outcomes that lacked explanation were always negative in nature (e.g., a camp counselor getting reprimanded). Given that people are particularly prone to seeking explanations for negative outcomes (e.g., Weiner, 1985), one important question for future research is to assess whether the valence of a witnessed outcome might mediate false memory for explanatory suggestions (cf., Chrobak, Groves, \& Otradovec, 2016). Second, an important, but as yet unstudied, issue is how a person's motivation to seek causal explanations (e.g., for highly consequential, unexpected, self-relevant outcomes) might influence how discerning a person will be when presented with potential suggested explanations (e.g., willingness to accept suggestions that are implausible or from an untrustworthy source). These remain important issues for future research.

\section{Implications}

The search for causal explanations is a basic human tendency that serves us well in many ways. For example, many of our most important discoveries in science, medicine, and technology were likely driven, at least in part, by the search for causal explanations. However, as evidenced by the findings reported here, the search for causal explanations also has a potential downside. Specifically, the present findings show that when people encounter false, or misleading information, the search for causal explanations can lead them astray, rendering them especially susceptible to developing false memories for misinformation that serves an explanatory function. Although the present study focuses on the specific problem of eyewitness suggestibility, these findings have implications that extend beyond the forensic context. Given the prevalence of misinformation in current society, and the many societal problems whose causes are so poorly understood, the potential for creating and spreading mistaken beliefs and memories is high.
Indeed, it may well be the case that many of the myths that pervade today's society (e.g., the unsubstantiated belief that vaccines cause autism) arose, at least in part, because they serve an explanatory function.

\section{References}

Ackil, J. K., \& Zaragoza, M. S. (1998). Memorial consequences of forced confabulation: Age differences in susceptibility to false memories. Developmental Psychology, 34, 1358-1372.

Ackil, J. K., \& Zaragoza, M. S. (2011). Forced fabrication versus interviewer suggestions: Differences in false memory depend on how memory is assessed. Applied Cognitive Psychology, 25(6), 933-942.

Blank, H., \& Launay, C. (2014). How to protect eyewitness memory against the misinformation effect: A meta-analysis of post-warning studies. Journal of Applied Research in Memory and Cognition, 3(2014), 77-88.

Chrobak, Q. M., \& Zaragoza, M. S. (2008). Inventing stories: Forcing witnesses to fabricate entire fictitious events leads to freely reported false memories. Psychonomic Bulletin \& Review, 15, 1190-1195.

Chrobak, Q. M., \& Zaragoza, M. S. (2013). When forced fabrications become truth: Causal explanations and false memory development. Journal of Experimental Psychology: General, 142(3), 827-844.

Chrobak, Q. M., Rindal, E. J., \& Zaragoza, M. S. (2015). The impact of multifaceted questions on eyewitness accuracy following forced fabrication interviews. The Journal of General Psychology, 142(3), 150-166.

Chrobak, Q. M., Groves, C. L., \& Otradovec, T. (2016). The impact of outcome valence on the susceptibility to suggestion for post-event causal misinformation. The Journal of General Psychology, 143(1), 16-32.

Einhorn, H. J., \& Hogarth, M. (1986). Judging probable cause. Psychological Bulletin, 99, 3-19.

Faul, F., Erdfelder, E., Lang, A. G., \& Buchner, A. (2007). G* Power 3: A flexible statistical power analysis program for the social, behavioral, and biomedical sciences. Behavior Research Methods, 39(2), 175-191.

Hanba, J. M., \& Zaragoza, M. S. (2007). Interviewer feedback in repeated interviews involving forced confabulation. Applied Cognitive Psychology, 21, 433-455.

Johnson, M. K., Hashtroudi, S., \& Lindsay, D. S. (1993). Source monitoring. Psychological Bulletin, 114(1), 3-28.

Kelley, H. H. (1973). The process of causal attribution. American Psychologist, 28, 107-128.

Lindsay, D. S. (2008). Source monitoring. In J. Byrne (Series Ed.) \& H.L. Roediger III (Vol. Ed.), Learning and memory: a comprehensive reference: Vol. 2. Cognitive psychology of memory (pp. 325-348). Oxford: Elsevier.

Lindsay, D., \& Johnson, S. (1989). The reversed eyewitness suggestibility effect. Bulletin of the Psychonomic Society, 27(2), 111-113.

Magliano, J. P., Radvansky, G. A., \& Copeland, D. E. (2007). Beyond language comprehension: Situation models as a form of autobiographical memory. In F. Schmalhofer \& C. A. Perfetti (Eds.), Higher level language processes in the brain: Inference and comprehension processes (pp. 379-391). Mahwah, NJ: Erlbaum.

McNamara, D. S., \& Magliano, J. (2009). Toward a comprehensive model of comprehension. In B. Ross (Ed.), The psychology of learning and motivation (Vol. 51, pp. 297-384). Burlington, MA: Academic Press.

Memon, A., Zaragoza, M., Clifford, B. R., \& Kidd, L. (2010). Inoculation or antidote? The effects of cognitive interview timing on false memories for forcibly fabricated events. Law and Human Behavior, 34, $105-117$. 
Schank, R. C., \& Abelson, R. (1977). Scripts, goals, plans, and understanding. Hillsdale, NJ: Erlbaum.

Weiner, B. (1985). Spontaneous" causal thinking. Psychological Bulletin, 97, 74-84.

Zacks, J. M., Speer, N. K., \& Reynolds, J. R. (2009). Segmentation in reading and film comprehension. Journal of Experimental Psychology: General, 138, 307-327.

Zaragoza, M. S., \& Lane, S. M. (1994). Source misattributions and the suggestibility of eyewitness memory. Journal of Experimental Psychology: Learning, Memory, and Cognition, 20, 934-945.
Zaragoza, M. S., Payment, K. E., Ackil, J. K., Drivdahl, S. B., \& Beck, M. (2001). Interviewing witnesses: Forced confabulation and confirmatory feedback increase false memories. Psychological Science, 12, 473-477.

Zaragoza, M. S., Belli, R. S., \& Payment, K. E. (2006). Misinformation effects and the suggestibility of eyewitness memory. In M. Garry \& H. Hayne (Eds.), Do justice and let the sky fall: Elizabeth F. Loftus and her contributions to science, law, and academic freedom (pp. 35-63). Hillsdale, NJ: Erlbaum.

Zwaan, R. A., \& Radvansky, A. (1998). Situation models in language comprehension and memory. Psychological Bulletin, 123, 162-185. 